\title{
White Matter
}

National Cancer Institute

\section{Source}

National Cancer Institute. White Matter. NCI Thesaurus. Code C33892.

The nerve tissue forming the bulk of the deep parts of the brain and the superficial parts of the spinal cord. It is composed of nerve cell processes, i.e. extensions (axons), which connect various grey matter areas of the brain to each other and carry nerve impulses to and from the nerve cell bodies within the central nervous system (neurons). Cerebral and spinal white matter does not contain dendrites. White matter is distinguished in that it is composed of axonal nerve fibers covered by a myelin sheath. 